\title{
Leishmania infantum in Tigers and Sand Flies from a Leishmaniasis- Endemic Area, Southern Italy
}

\author{
Roberta latta, Andrea Zatelli, Pietro Laricchiuta, Matteo Legrottaglie, \\ David Modry, Filipe Dantas-Torres, Domenico Otranto
}

We detected Leishmania infantum infection in $45 \%$ of tigers and $5.3 \%$ of sand flies tested at a zoo in southern Italy in 2019. These infections in tigers and the abundance of Phlebotomus perniciosus sand flies represent a potential risk to other animals and humans living in or visiting the zoo.

$\mathrm{V}^{\mathrm{i}}$ isceral leishmaniasis, caused by infection with Leishmania infantum protozoa, is listed among the most neglected tropical diseases, affecting thousands of persons, most of whom are among the world's most vulnerable populations (1). The disease is associated with the presence of phlebotomine sand fly vectors; domestic dogs typically act as reservoirs. Among felids, domestic cats have recently gained prominence as putative reservoirs of L. infantum (2), whereas cases of infection in other felids have been reported occasionally (3-5).

In February 2019, a tiger (index case), born and raised in a zoologic park in southern Italy, had a nonhealing laceration that tested positive for L. infantum DNA on a skin punch biopsy. Because tigers are considered an endangered species, the presence of an active L. infantum transmission focus in a facility visited by thousands of visitors each year deserves attention. Therefore, we conducted an epidemiologic study to investigate the prevalence of L. infantum infection in the local tiger and sand fly populations, along with the sand flies' host blood-feeding preferences.

Author affiliations: University of Bari, Bari, Italy (R. latta, A. Zatelli, F. Dantas-Torres, D. Modry, D. Otranto); Zoo Safari di Fasano, Brindisi, Italy (P. Laricchiuta, M. Legrottaglie); University of Veterinary and Pharmaceutical Sciences, Brno, Czech Republic (D. Modry); Czech Academy of Sciences, Ceske Budejovice, Czech Republic (D. Modry); Masaryk University, Brno (D. Modry); Fundação Oswaldo Cruz (Fiocruz), Recife, Brazil (F. Dantas-Torres); Bu-Ali Sina University, Hamedan, Iran (D. Otranto).

DOI: https://doi.org/10.3201/eid2606.191668

\section{The Study}

During March-June 2019, we tested 20 tigers born at the zoologic park (Safari Park, Apulia region, Brindisi Province, southern Italy) and living in an open enclosure for L. infantum infection. We smeared lymph node aspirates on slides for the cytologic examination; we also cultured and processed these specimens, along with whole blood, skin punch biopsy, and conjunctival, nasal and oral swab specimens, for the detection of L. infantum DNA by quantitative PCR (qPCR) (6). We tested for feline leukemia virus (FeLV) and feline immunodeficiency virus (FIV) by using proviral DNA from blood, as described previously (7). We detected L. infantum antibodies by using an immunofluorescence antibody test (IFAT), as described previously in a study in cats (2). During May-November 2019, we collected sand flies in the tigers' enclosure biweekly by using sticky traps and light traps and identified each specimen by using morphologic keys. We performed conventional PCR for blood-meal identification in sand flies by using primers cyto 1 (5'-CCATCAAACATCTCAGCATGAAA-3') and T2893R (5'-GTTGGCGGGGATGTAGTTATC-3'), which target the mitochondrial cytochrome $b$. The protocol of this study was approved by the ethics committee of the Department of Veterinary Medicine at the University of Bari (Bari, Italy).

Tigers enrolled in the study ranged in age from 6 months to 11 years and weighed $70-220 \mathrm{~kg}$ (Table 1); all were apparently healthy or had unrelated conditions, except for 1 (index case), which had a large nonhealing laceration extending from the left loin region to the left thoracic region (Figure). Overall, 9 (45\%) of the 20 tigers tested positive for L. infantum by IFAT, $5(25 \%)$ tested positive by qPCR, and $5(25 \%)$ tested positive by both methods (Table 1). The tigers were positive by qPCR on lymph node aspirates and skin punch biopsy. None of the conjunctival swab specimens tested positive. We did not detect L. infantum 
Table 1. Serologic and molecular results for Leishmania infantum in 20 tigers, southern Italy*

\begin{tabular}{|c|c|c|c|c|c|c|c|c|c|c|}
\hline \multirow{2}{*}{$\begin{array}{l}\text { Month of sample } \\
\text { collection }\end{array}$} & \multirow[b]{2}{*}{ Case no. } & \multirow{2}{*}{$\begin{array}{l}\text { Age, } \\
\text { y/sex }\end{array}$} & \multirow[b]{2}{*}{ Weight, kg } & \multirow[b]{2}{*}{ IFAT/Ab titer } & \multicolumn{6}{|c|}{ qPCR results } \\
\hline & & & & & PB & LN & CS & NS & OS & SPB \\
\hline March & 1 & $7 / \mathrm{F}$ & 150 & $1: 160$ & - & + & - & - & - & + \\
\hline April & 2 & $6 / \mathrm{M}$ & 160 & $1: 80$ & - & NT & - & - & - & - \\
\hline April & 3 & $7 / M$ & 220 & $1: 80$ & - & - & - & - & - & - \\
\hline April & 4 & $8 / \mathrm{M}$ & 210 & $1: 80$ & - & NT & - & + & + & - \\
\hline April & 5 & $7 / \mathrm{M}$ & 220 & - & - & - & - & - & - & - \\
\hline April & 6 & 9/F & 150 & - & - & - & - & - & - & - \\
\hline May & 7 & $2 / F$ & 135 & $1: 640$ & - & + & - & + & - & + \\
\hline May & 8 & $2 / \mathrm{M}$ & 180 & - & - & NT & - & - & - & - \\
\hline May & 9 & $2 / F$ & 150 & $1: 40$ & + & + & - & - & - & + \\
\hline May & 10 & $2 / \mathrm{M}$ & 170 & $1: 40$ & + & + & - & - & + & + \\
\hline May & 11 & $8 / F$ & 190 & $1: 40$ & - & - & - & - & - & - \\
\hline May & 12 & $6 / M$ & 220 & - & - & - & - & - & - & - \\
\hline May & 13 & $7 / \mathrm{F}$ & 160 & - & - & - & - & - & - & - \\
\hline May & 14 & $11 / F$ & 120 & - & - & - & - & - & - & - \\
\hline June & 15 & $1 / \mathrm{M}$ & 130 & - & - & - & - & - & - & - \\
\hline June & 16 & $1 / F$ & 110 & - & - & - & - & - & - & - \\
\hline June & 17 & $7 / F$ & 150 & 1:80 & - & - & - & - & - & - \\
\hline June & 18 & $0.5 / F$ & 70 & - & - & - & - & - & - & - \\
\hline June & 19 & $0.5 / F$ & 70 & - & - & - & - & - & - & - \\
\hline June & 20 & $0.5 / F$ & 70 & - & - & - & - & - & - & - \\
\hline
\end{tabular}

${ }^{*}$ Ab, antibody; CS, conjunctival swab; IFAT, immunofluorescence antibody test; LN, lymph node; NS, nasal swab; NT, not tested; OS, oral swab; PB, peripheral blood; qPCR, quantitative PCR; SPB, skin punch biopsy; -, negative; +, positive.

cytology or culture of lymph node aspirates in any of the tigers. All tigers were negative for FeLV and FIV.

During May-November 2019, we collected a total of 580 sand flies. The most abundant species was Phlebotomus perniciosus ( $\mathrm{n}=491)$, followed by Sergentomyia minuta $(\mathrm{n}=69)$ and $P$. neglectus $(\mathrm{n}=20)$. Of the 190 females collected, 151 (26\%) were P. perniciosus, 4 $(<1 \%)$ were $P$. neglectus, and $35(6 \%)$ were S. minuta. Specimens for $8(5.3 \%)$ P. perniciosus sand flies and 1 (2.9\%) S. minuta sand fly tested positive for L. infantum DNA. Of the 190 females examined, 63 (33.1\%) P. perniciosus, 3 (1.6\%) P. neglectus, and 2 (1.1.\%) S. minuta sand flies tested positive for tiger DNA (Table 2); we detected no other mammalian DNA (e.g., from cats, dogs, rats, or humans) in blood-fed or -unfed specimens.

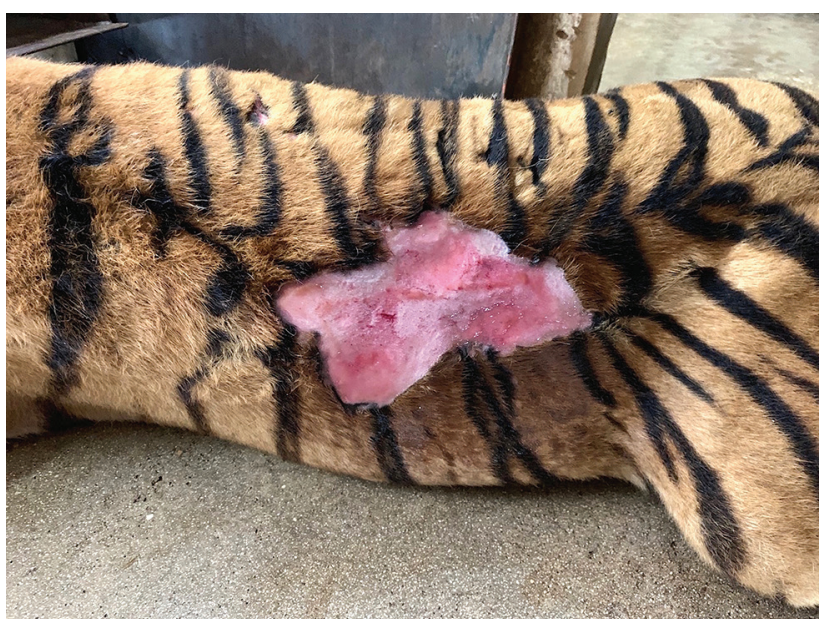

Figure. Large nonhealing laceration, attributable to Leishmania infantum infection, extending from the left loin region to the left thoracic region of a tiger, southern Italy.
Consensus sequences of the vertebrate host mitochondrial cytochrome $b$ from all female sand flies (positive specimens) displayed $100 \%$ identity to the nucleotide sequences of Panthera tigris available in the GenBank database (accession nos. MH124112 and KC879295).

\section{Conclusions}

The high prevalence $(45 \%)$ of L. infantum infection recorded indicates that tigers living in the zoologic park are highly exposed to sand flies and thus have a high risk for acquiring the parasite. The finding of engorged sand flies that fed on tigers and were also positive for L. infantum suggest that tigers could be an alternative host of this parasite; however, the possibility that L. infantum-positive sand flies had acquired the infection from another host, before feeding on tigers, cannot be ruled out.

Although Leishmania spp. infection has been scantly described in wild felids (3-5), the diagnosis of this parasitic infection should also be considered while screening these animals for pathogens potentially impairing their health and welfare. No information is available on the immune response against $L$. infantum infection in tigers, and serologic tests have not been validated for this host, but one could reasonably suspect that their antibody production would follow a pattern similar to that occurring in cats. Nonetheless, the absence of L. infantum DNA in tigers that were positive for L. infantum antibodies (4/9 tigers [44.4\%]) could be expected, given that this lack of correlation between molecular and serologic positivity has also been observed in cats (2), indicating that the diagnosis of the infection in these animals might 
Table 2. Number of phlebotomine sand fly species by sex, positivity for Leishmania infantum by quantitative PCR, and blood meal on tigers, southern Italy*

\begin{tabular}{|c|c|c|c|c|c|c|}
\hline \multirow[b]{2}{*}{ Sand fly species } & \multicolumn{2}{|c|}{ Sex } & \multirow[b]{2}{*}{ Total } & \multirow{2}{*}{$\begin{array}{l}\text { No. }(\%) \text { positive } \\
\text { for L. infantum }\end{array}$} & \multicolumn{2}{|c|}{ Blood meal source } \\
\hline & $\mathrm{F}$ & $\mathrm{M}$ & & & No. positive & No. positive/engorged \\
\hline Phlebotomus perniciosus & 151 & 340 & 491 & $8 / 151(5.3)$ & $63 / 151$ & $48 / 63$ \\
\hline Sergentomyia minuta & 35 & 34 & 69 & $1 / 35(2.9)$ & $2 / 35$ & - \\
\hline Phlebotomus neglectus & 4 & 16 & 20 & - & $3 / 4$ & - \\
\hline Total & 190 & 390 & 580 & - & $68 / 190$ & $48 / 63$ \\
\hline
\end{tabular}

be a difficult task, as it is in cats. The detection of $L$. infantum DNA in the lymph node aspirate and skin biopsy suggests that these tissues are more suitable than blood for the diagnosis of this infection, as previously reported in dogs and cats $(8,9)$. Otherwise, the conjunctival swab seems to be not as good a sample for this purpose in tigers. Unlike some studies with cats (2), no correlation between L. infantum infection and FIV, FELV, or both FIV and FELV infection has been observed in the tigers in our study.

The predominance of $P$. perniciosus sand flies, along with their positivity for L. infantum DNA already recorded in southern Italy $(10,11)$, is somewhat expected, given that this sand fly species is recognized as the main vector for $L$. infantum in different foci of visceral leishmaniasis in Italy (12). The high proportion of L. infantum-infected sand flies suggests that the risk for parasite transmission in this environment should be considered. Furthermore, the detection of L. infantum DNA in S. minuta sand flies has already been reported in southern Italy $(4.2 \%)$ and Portugal $(4 \%)(11,13)$. In addition, although consideration of the role played by $S$. minuta (the proven vector of $L$. tarentolae) in the circulation of Leishmania spp. of zoonotic concern has been raised $(11,14)$, further studies are necessary to fully assess its vector role.

P. perniciosus sand flies frequently feed on tigers, because dogs are not allowed to roam in the zoo, the role of tigers as local reservoir hosts needs to be ascertained. Because P. perniciosus sand flies feed on a wide range of domestic and wild animals, and because $L$. infantum might infect the sand flies after taking a blood meal from infected felids (15), the role of tigers in the transmission cycle of L. infantum is probable.

In summary, L. infantum infection should be included in the differential diagnosis of infectious diseases in tigers in areas where visceral leishmaniasis is endemic. The role of tigers as sentinels for L. infantum, the occurrence of $P$. perniciosus sand flies infected by the protozoan, and its abundance in the study area might represent an eminent risk for animals and humans living in or visiting the zoo. Therefore, prevention measures are needed for providing protection against $L$. infantum infection in these animals and for controlling sand flies.

\section{About the Author}

Dr. Iatta is an associate professor at the Department of Veterinary Medicine, University of Bari "Aldo Moro" in Italy. Her research interests include the diagnosis, epidemiology, and prevention of parasitic infectious diseases.

\section{References}

1. World Health Organization. Leishmaniasis 2018 [cited 2018 Dec 22]. https://www.who.int/leishmaniasis

2. Iatta R, Furlanello T, Colella V, Tarallo VD, Latrofa MS, Brianti E, et al. A nationwide survey of Leishmania infantum infection in cats and associated risk factors in Italy. PLoS Negl Trop Dis. 2019;13:e0007594. https://doi.org/10.1371/ journal.pntd.0007594

3. de Oliveira AR, de Carvalho TF, Arenales A, Tinoco HP, Coelho CM, Costa MELT, et al. Mandibular squamous cell carcinoma in a captive Siberian tiger (Panthera tigris altaica). Braz J Vet Pathol. 2018;11:97-101. https:/ / doi.org/10.24070/ bjvp.1983-0246.v11i3p97-101

4. Libert C, Ravel C, Pratlong F, Lami P, Dereure J, Keck N. Leishmania infantum infection in two captive barbary lions (Panthera leo leo). J Zoo Wildl Med. 2012;43:685-8. https://doi.org/10.1638/2012-0056.1

5. Dahroug MA, Almeida AB, Sousa VR, Dutra V, Turbino NC, Nakazato L, et al. Leishmania (Leishmania) chagasi in captive wild felids in Brazil. Trans R Soc Trop Med Hyg. 2010;104:73-4. https:// doi.org/10.1016/j.trstmh.2009.08.003

6. Francino O, Altet L, Sánchez-Robert E, Rodriguez A, Solano-Gallego L, Alberola J, et al. Advantages of real-time PCR assay for diagnosis and monitoring of canine leishmaniosis. Vet Parasitol. 2006;137:214-21. https://doi.org/10.1016/j.vetpar.2006.01.011

7. Stiles J, Bienzle D, Render JA, Buyukmihci NC, Johnson EC. Use of nested polymerase chain reaction (PCR) for detection of retroviruses from formalin-fixed, paraffinembedded uveal melanomas in cats. Vet Ophthalmol. 1999;2:113-6. https://doi.org/10.1046/j.1463-5224.1999.00066.x

8. Pennisi MG, Lupo T, Malara D, Masucci M, Migliazzo ALG. Serological and molecular prevalence of Leishmania infantum infection in cats from southern Italy. J Feline Med Surg. 2012;14:656-7.

9. Otranto D, Paradies P, de Caprariis D, Stanneck D, Testini G, Grimm F, et al. Toward diagnosing Leishmania infantum infection in asymptomatic dogs in an area where leishmaniasis is endemic. Clin Vaccine Immunol. 2009;16:337-43. https:// doi.org/10.1128/CVI.00268-08

10. Tarallo VD, Dantas-Torres F, Lia RP, Otranto D. Phlebotomine sand fly population dynamics in a leishmaniasis endemic peri-urban area in southern Italy. Acta Trop. 2010;116:227-34. https://doi.org/10.1016/ j.actatropica.2010.08.013

11. Latrofa MS, Iatta R, Dantas-Torres F, Annoscia G, Gabrielli S, Pombi M, et al. Detection of Leishmania infantum 
DNA in phlebotomine sand flies from an area where canine leishmaniosis is endemic in southern Italy. Vet Parasitol. 2018;253:39-42. https:// doi.org/10.1016/j.vetpar.2018.02.006

12. Maroli M, Feliciangeli MD, Bichaud L, Charrel RN, Gradoni L. Phlebotomine sandflies and the spreading of leishmaniases and other diseases of public health concern. Med Vet Entomol. 2013;27:123-47. https://doi.org/10.1111/ j.1365-2915.2012.01034.x

13. Pereira S, Pita-Pereira D, Araujo-Pereira T, Britto C, Costa-Rego T, Ferrolho J, et al. First molecular detection of Leishmania infantum in Sergentomyia minuta (Diptera, Psychodidae) in Alentejo, southern Portugal. Acta Trop. 2017;174:45-8. https://doi.org/10.1016/j.actatropica. 2017.06.020
14. Maia C, Depaquit J. Can Sergentomyia (Diptera, Psychodidae) play a role in the transmission of mammalinfecting Leishmania? Parasite. 2016;23:55. https:// doi.org/ 10.1051/parasite/2016062

15. Maroli M, Pennisi MG, Di Muccio T, Khoury C, Gradoni L, Gramiccia M. Infection of sandflies by a cat naturally infected with Leishmania infantum. Vet Parasitol. 2007;145:357-60. https://doi.org/10.1016/j.vetpar.2006.11.009

Address for correspondence: Domenico Otranto, Department of Veterinary Medicine, University of Bari, Strada Provinciale per Casamassima km 3, 70010, Valenzano (BA) 70010, Italy; email: domenico.otranto@uniba.it

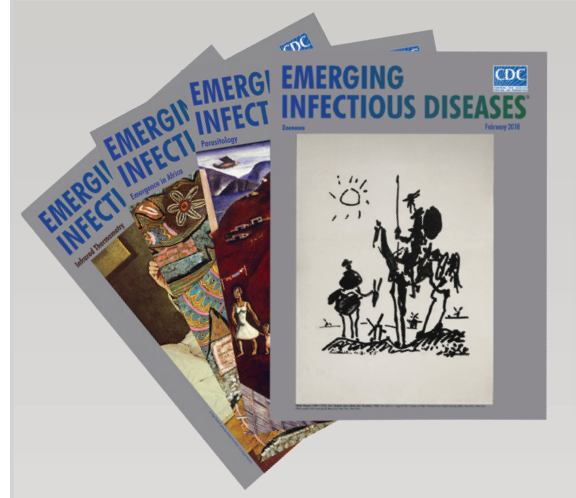

- Increase in Ocular Syphilis Cases at Ophthalmologic Reference Center, France, 2012-2015

- Adenovirus Type 4 Respiratory Infections among Civilian Adults, Northeastern United States, 2011-2015

- Ecologic Features of Plague Outbreak Areas, Democratic Republic of the Congo, 2004-2014

- Hypervirulent Klebsiella pneumoniae in Cryptogenic Liver Abscesses, Paris, France

- Echinococcus spp. Tapeworms in North America

- Borrelia miyamotoi Infections in Humans and Ticks, Northeastern China

- Plasmid-Encoded Transferable mecB-Mediated Methicillin Resistance in Staphylococcus aureus

- Multiplex PCR-Based Next-Generation Sequencing and Global Diversity of Seoul Virus in Humans and Rats

- Clinical and Molecular Epidemiology of Staphylococcal Toxic Shock Syndrome in the United Kingdom

- Lethal Respiratory Disease Associated with Human Rhinovirus C in Wild Chimpanzees, Uganda, 2013

- Spread of Meropenem-Resistant Streptococcus pneumoniae Serotype 15A-ST63 Clone in Japan, 2012-2014

\section{February 2018}

\section{Zoonoses}

- Role of Environmental Factors in Shaping Spatial Distribution of Salmonella enterica Serovar Typhi, Fiji

- Yersinia pestis Survival and Replication in Potential Ameba Reservoir

- New Parvovirus Associated with Serum Hepatitis in Horses after Inoculation of Common Biological Product

- Development of a Pediatric Ebola Predictive Score, Sierra Leone

- Trends in Infectious Disease Mortality, South Korea, 1983-2015

- Use of Pristinamycin for Macrolide-Resistant Mycoplasma genitalium Infection

- Risk Communication and Ebola-Specific Knowledge and Behavior during 2014-2015 Outbreak, Sierra Leone

- Macacine Herpesvirus 1 Antibody Prevalence and DNA Shedding among Invasive Rhesus Macaques, Silver Springs State Park, Florida, USA

- Co-circulation of Influenza A H5, H7, and H9 Viruses and Co-infected Poultry in Live Bird Markets, Cambodia

- Scrub Typhus Outbreak in Chonburi Province, Central Thailand, 2013 